\title{
Backscattering spectroscopy for assessing skin tumor
}

\author{
Valery P. Zakharov ${ }^{1}$, Pavel E. Timchenko ${ }^{1 *}$, Elena V. Timchenko ${ }^{1}$, Larisa A. Zherdeva ${ }^{1}$, \\ Sergey. V. Kozlov ${ }^{2}$, Alexander A. Moryatov ${ }^{2}$ \\ ${ }^{1}$ Samara State Aerospace University (SSAU), 34 Moskovskoye Shosse, Samara 443086, Russia \\ ${ }^{2}$ Samara State Medical University, 89 Chapaevskaya str., Samara 443099, Russia \\ *e-mail: timpavel@mail.ru
}

\begin{abstract}
The results of in vitro backscattering spectral studies of 7 nevuses and 27 samples of different skin cancer are presented. The pathology type classification in the two-dimensional phase space formed by the scattering indices at the wavelengths 560 $\mathrm{nm}, 650 \mathrm{~nm}, 700 \mathrm{~nm}$, and $760 \mathrm{~nm}$ scaled to the healthy skin scattering coefficient value is proposed. It is shown that the sensitivity and specificity of differentiation between the benign and malignant tumors exceeds $86 \%$ and $96 \%$, respectively, and their value for selecting melanoma among other malignant tumors falls by $5-8 \%$ due to partial overlap of classes in the phase space. (C) 2015 Samara State Aerospace University (SSAU).
\end{abstract}

Keywords: Differential backscattering, discriminant analysis, tumor, noninvasive diagnostics, optical coefficient, melanoma, basal cell carcinoma, nevus.

Paper \#2489 received 2015.05.05; revised manuscript received 2015.06.19; accepted for publication 2015.06.25; published online 2015.06.30.

\section{References}

1. L. V. Wang, "Skin cancer detection by spectroscopic oblique-incidence reflectometry: classification and physiological origins," Appl. Opt. 43, 2643-2650 (2004).

2. A. V. Novik, "Skin melanoma: novel approaches," Prakticheskaya onkologiya 1(12), 36-42 (2011).

3. M. Mogensen, and G. B. Jemec, "Diagnosis of nonmelanoma skin cancer/keratinocyte carcinoma: a review of diagnostic accuracy of nonmelanoma skin cancer diagnostic tests and technologies," Dermatol Surg 33(10), 1158-1174 (2007).

4. S. W. Fosko, W. Hu, T. F. Cook, et al., "Positron emission tomography for basal cell carcinoma of the head and neck," Arch Dermatol 139, 1141-1146 (2003).

5. V. P. Zakharov, K. V. Larin, and I. A. Bratchenko, "Increasing the informativity of optical coherence tomography in skin pathology diagnistics," Proceedings of SSAU 2(26), 232-239 (2011).

6. A. Alex, J. Weingast, B. Hofer, M. Eibl, M. Binder, H. Pehamberger, W. Drexler, and B. Povazay, "3D optical coherence tomography for clinical diagnosis of nonmelanoma skin cancers," Imaging Med 6, 653-674 (2011).

7. A. Scope, U. Mahmood, D. S. Gareau, M. Kenkre, J. A. Lieb, K. S. Nehal, M. Rajadhyaksha, "In vivo reflectance confocal microscopy of shave biopsy wounds: feasibility of intraoperative mapping of cancer margins," Br J Dermatol 163, 1218-1228 (2010).

8. J. Zhao, H. Lui, D. I. McLean, and H. Zeng, "Real-time Raman spectroscopy for noninvasive in vivo skin analysis and diagnosis," New developments in biomedical engineering 24, 455-474 (2010).

9. A. Garcia-Uribe, J. Zou, M. Duvic, J. H. Cho-Vega, V. G. Prieto, and L. V. Wang, "In Vivo Diagnosis of Melanoma and Nonmelanoma Skin Cancer Using Oblique Incidence Diffuse Reflectance Spectrometry," Cancer Res 72(11), 2738-2745 (2012).

10. J. de Leeuw, N. van der Beek, W. D. Neugebauer, P. Bjerring, and H. A. Neumann, "Fluorescence detection and diagnosis of non-melanoma skin cancer at an early stage," Lasers Surg Med 41(2), 96-103 (2009).

11. V. P. Zakharov, I. A. Bratchenko, O. O. Myakinin, D. N. Artemyev, D. V. Kornilin, S. V. Kozlov, and A. A. Moryatov, "Multimodal diagnostics and imaging of oncological pathologies," Quantum Electron 44(8), 726731 (2014).

12. A. Pradhan, B. B. Das, K. M. Yoo, R. R. Alfano, J. Cleary, R. Prudente, and E. Celmer, "Time-resolved UV photoexcited fluorescence kinetics from malignant and non-malignant breast tissues," Proc. SPIE Conference 1599, 81-84 (1992). 
13. C. Y. Wang, H. M. Chen, C. P. Chiang, C. You, and T. C. Hsiao, "Time-resolved autofluorescence spectroscopy for classifying normal and premalignant oral tissues," Lasers Surg. Med 37(1), 37-45 (2005).

14. P. V. Butte, B. K. Pikul, A. Hever, W. H. Yong, K. L. Black, and L. Marcu, "Diagnosis of meningioma by time resolved fluorescence spectroscopy," J. Biomed. Opt 10(6), 064026 (2005).

15. N. P. Galletly, J. Mcginty, C. Dunsby, F. Teixeira, J. Requejo-Isidro, I. Munro, D. S. Elson, M. A. A. Neil et al., "Fluorescence lifetime imaging distinguishes basal cell carcinoma from surrounding uninvolved skin," Br. J. Dermatol 159(1), 152-161 (2008).

16. J. Mcginty, N. P. Galletly, C. Dunsby, I. Munro, D. S. Elson et al., "Wide-field fluorescence lifetime imaging of cancer," Biomed. Opt. Express 1(2), 627-640 (2010).

17. R. Cicchi, A. Crisci, A. Cosci, G. Nesi, D. Kapsokalyvas, S. Giancane, M. Carini, and F. S. Pavone, "Timeand Spectral-resolved two-photon imaging of healthy bladder mucosa and carcinoma in situ," Opt. Express 18(4), 3840-3849 (2010).

18. X. He, K. Wang, and Z. Cheng, "In vivo near-infrared fluorescence imaging of cancer with nanoparticle-based probes," Nanomed Nanobiotechnol 2, 349-366 (2010).

19. G. Wagnieres, J. Mizeret, A. Strudzinski, and H. Van den Bergh, "Frequence-domain fluorescence lifetime imaging for endoscopic clinical cancer photodetection: apparatus design and preliminary results," J. Fluoresc. 7, 75-83 (1997).

20. A. Garcia-Uribe, E. B. Smith, M. Duvic, and L. V. Wang, "White light oblique-incidence diffuse reflectance spectroscopy for classification of in vivo pigmented skin lesions," Proc. SPIE 6435, 64350L (2007).

21. I. Kuzmina, I. Diebele, D. Jakovels, J. Spigulis, L. Valeine, J. Kapostinsh, and A. Berzina, "Towards noncontact skin melanoma selection by multi-spectral imaging analysis," J. Biomed. Opt. 16(6), 1-3 (2011)

22. A. J. Thompson et.al., "In vivo measurements of diffuse reflectance and time-resolved autofluorescence emission spectra of basal cell carcinomas," J. Biophotonics 5(3), 240-254 (2012).

23. P. J. Matts, P. J. Dykes, and R. Marks, "The distribution of melanin in skin determined in vivo, British Association of Dermatologists," British Journal of Dermatology 156, 620-628 (2007).

24. G. Z. A. Dimou, I. Bassukas, D. Galaris, A. T. E. Kaxiras, "Melanin absorption spectroscopy: new method for noninvasive skin investigation and melanoma detection," Journal of Biomedical Optics 13(1), 014017 (2008).

25. R. O. Duda, P. E. Hart, and D. G. Stork, Pattern Classification, 2nd Edition, Wiley (2000).

26. M. Mogensen et al., "Optical coherence tomography for imaging of skin and skin diseases," Semin. Cutan. Med. Surg. 28, 196-202 (2009).

\section{Introduction}

The intensified impact of technology factors on man, the worsening of ecologic conditions, and the increase of the carcinogen air pollution lead to the growth of the number of skin oncologic diseases among the population, namely, basal cell carcinoma, squamous cell carcinoma and melanoma [1]. The latter kind of tumor is the most dangerous one, since its appearance is like that of the benign tumor (nevus), and the lately detected melanoma is hardly treatable and often is accompanied with the development of metastases. At early melanoma stages the depth of invasion is insignificant and the efficiency of its treatment is essentially higher [2].

A commonly accepted method of the tumor type assessment is the histologic analysis of a tissue sample taken for biopsy. In application to the skin oncologic diseases the histology is performed after the surgical operation and, therefore, the unambiguous information about the tumor type is absent at the moment of operation. This leads to the increased amount of surgical intervention due to the uncertain invasion depth, as well as to excess surgical operations of purely benign tumors [3].

In this connection it is an urgent problem to develop methods for high-efficiency express diagnostics of the neoplasm kind during the pre-surgery period, which can essentially reduce the numbers of recurrent tumours. For the noninvasive skin cancer diagnostics the application of biomedical imaging is known, such as PET [4], optical coherence tomography $[5,6]$, confocal microscopy [7]. However, the imaging methods are labour-consuming and expensive, and their application is reasonable for updating the already established diagnosis, planning and control of the treatment process.

The screening of skin oncologic diseases is most convenient using optical methods, e.g., Raman scattering [8], backscattering [9], and fluorescence analysis [10]. Due to small cross section the Raman scattering requires long signal acquisition time for random noise elimination, which hampers its mass application without combining with optical imaging methods [11]. As a rule, the fluorescence is excited with UV radiation [12, 13], because the strongest absorption lines of natural fluorochromes are in the UV region. The reliability of fluorescence diagnostics can be improved by using either the complicated methods of timeresolved fluorescence spectroscopy [14-16], or the multiphoton fluorescence analysis [17] using laser sources with high peak power of probing radiation. An alternative method of improving the fluorescence analysis efficiency is the gold nanoparticles injection [18]. Having a number of positive properties, the fluorescence methods suffer from limitations, they are unable to determine the true boundaries of the tumour 
with enough precision and to assess the condition of perifocal zones surrounding the tumour without injection of artificial fluorochromes [19].

The application of reflection spectroscopy to the study of skin spectral properties [20] demonstrated the possibility to use it for the assessment of malignant and benign skin tumors [21, 22]. It was shown that the spectral intensity of backscattering essentially differs for the basal cell carcinoma and the adjacent healthy skin at the wavelengths 540 and $575 \mathrm{~nm}$, corresponding to the haemoglobin absorption bands. This is due to the enhancement of capillary network in the tumor. This fact allowed to formulate a method for basal cell carcinoma differentiation based on the difference in spectral properties of the scattered radiation [22]. However, due to high variability of skin spectral properties in different patients $[20,23]$ the sensitivity of this method is insufficient for screening examination. The authors of Refs. [21, 24] proposed to use the difference in melanin absorption in the visible and near infrared spectral regions for the melanoma test. However, this approach does not allow differentiation of malignant melanoma from the benign nevus, since both are characterised by the increased melanin concentration.

The goal of the present paper is to develop the backscattering method for differentiation of tumors with the individual human skin features taken into account.

\section{Experimental technique}

The object of study were 27 samples of skin malignant tumors (12 melanomas, 5 samples of squamous cell carcinoma, and 10 samples of basal cell carcinoma) and 7 samples of benign skin tumor (pigment nevus). All samples were obtained after surgical resection in the State Health Institution Samara Regional Oncology Clinic (SHI SROC) and investigated according to the protocol, approved by the Bioethics Committee of Samara State Medical University. The tissue samples were kept in sterile boxes at $+4 \pm 2{ }^{\circ} \mathrm{C}$ and were tested not later than in four hours after resection. Each tumour examination was accompanied by histologic analysis for the final diagnosis. Alongside with the tumour region, in each sample the optical properties of healthy skin adjacent to the tumor region were also studied.

The registration and spectral analysis of radiation, scattered from the sample, were carried out using the experimental setup presented in Fig. 1. The broadband optical radiation, generated by the source 1, was supplied via the coupler 2 to the input 3 of the first receiver channel of the spectrometer 4 and via the fiber 5 onto the studied object 6 . The light scattered from the sample 6 was collected and supplied via the fiber 7 to the second receiver channel of the spectrometer 4 . The sample was attached to the microtransporter 8 with linear step motor 9. The control of the setup was performed from the personal computer 10 .

The radiator 1 generated optical radiation in the spectral range from 300 to $1100 \mathrm{~nm}$ with the intensity maximum at $750 \mathrm{~nm}$. The spectrograph 4 was the six- channel Shamrock SR-303i (focal length $303 \mathrm{~mm}$ ) with integrated digital camera ANDOR DV-420A-OE $(1024 * 256,26 \mu \mathrm{m} 2,16 \mathrm{bit})$, which provided the registration of the scattered radiation spectrum with the error $\pm 0.2 \mathrm{~nm}$ in the entire spectral range of the radiation source. The fibers 5 and 7 were fastened at the distance $1 \mathrm{~mm}$ from each other and formed a singe fiber-optical unit placed at the height of $1 \mathrm{~mm}$ above the sample 6. The height was determined experimentally to provide the maximal backscattering signal intensity. The diameter of the light spot at the sample 6 for this height was about $1 \mathrm{~mm}$. The use of microtransporter 8 allowed scanning the region of interest with the step 1 $\mathrm{mm}$ and the linear translation velocity $2 \mathrm{~mm} / \mathrm{s}$. This allowed rapid recording of the biotissue optical properties at $16-25$ points of the object of interest.

Simultaneous registration of incident (reference) and scattered (measuring channels 3 and 7 of the spectrometer 4) signal intensity allowed the elimination of power fluctuations of the source 2 and the thermal drift of its spectrum. For this aim at each moment of time the normalisation of the spectral density of scattered radiation to the spectral density recorded in the reference channel was carried out.

All experiments were carried out under similar temperature conditions, room illumination and setup geometry. To increase the signal-to-noise ratio the signal was accumulated during $0.2 \mathrm{~s}$ and smoothed using the median and averaging filters.

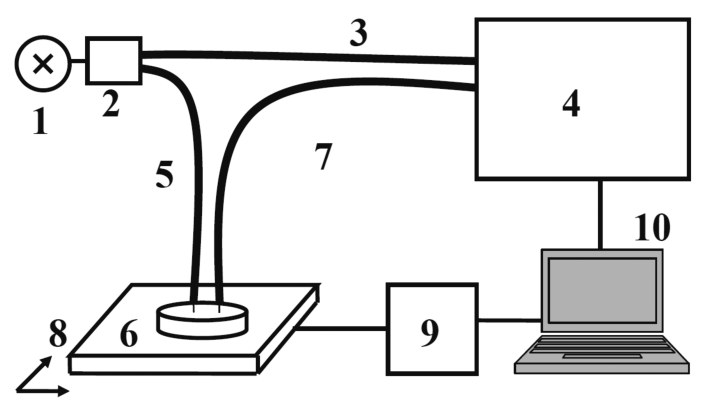

Fig. 1 Schematic diagram of the experimental setup: $1-$ broadband radiation source, 2 -coupler, 3 - reference signal optical fiber, 4 - multichannel spectrometer, 5 radiating fiber, 6 - object of study, 7 - diagnostic fiber, 8 - transporter, 9- step motor, 10 - computer.

\section{Spectral differentiation of skin tumors}

Let us introduce the scattering indices to characterise the skin optical properties at the point $(\mathrm{x}, \mathrm{y})$

$$
\begin{aligned}
& k(x, y)=\frac{I_{760}(x, y)}{I_{560}(x, y)}, \\
& m(x, y)=\lg \left(I_{650}(x, y)\right)-\lg \left(I_{700}(x, y)\right),
\end{aligned}
$$

where $I_{\lambda}(x, y)$ is the intensity of scattering at the point $(x, y)$ scaled to the intensity of the radiation source at the wavelength $\lambda$. 
In fact the index $k$ is related to the density of capillary network in dermis in the region of backscattering registration and the index $\mathrm{m}$ is proportional to the melanin concentration. As it is known [21], the tumor growth is accompanied by intense formation of capillary network that changes the skin optical properties in the region of tumor in the spectral regions of minimal and maximal haemoglobin absorption (the wavelengths $760 \mathrm{~nm}$ and $560 \mathrm{~nm}$, respectively). And in the melanoma or nevus case the increase of the melanin concentration manifests itself in exponential decrease of the intensity in the spectral range 560-700 $\mathrm{nm}$. In order to exclude the influence of hemoglobin content variations on melanin index $\mathrm{m}$ we use only near IR part of the range between wavelengths $650 \mathrm{~nm}$ and $700 \mathrm{~nm}$.

On the other hand, the optical properties of the skin are individual, and the concentration of melanin and other pigments essentially changes depending on the skin type. In the experiments carried out the samples belonged to the skin type II - light (light-skin Europeans) and III - medium-light (dark European). To compensate the difference in coefficients (1) the coefficients measured in the tumour were scaled to the average value of these coefficients for normal skin

$$
K(x, y)=\frac{k(x, y)}{\overline{\overline{k_{\text {norm }}}}}, M(x, y)=\frac{m(x, y)}{\overline{m_{\text {norm }}}}
$$

The averaged values of the coefficients $k_{\text {norm }}$ and $m_{\text {norm }}$ for the healthy skin were determined at 3-5 points located near the tumour at the distance $5-10 \mathrm{~mm}$ from its visible boundary.

Each measurement of the backscattered spectral intensity at the point $(x, y)$ of the tumour can be presented by a point in the phase plane $(K, M)$. Then the points located beyond the tumour will cluster about the centre with coordinates $(1,1)$ in the phase plane, and the rest space corresponds to the tumor region.

In Fig. 2 each studied sample is presented by the point $\left(K_{\max }, M_{\max }\right)$ that corresponds to the maximal deviation of the coefficients (2) from their averaged values in normal skin, i.e., from the phase point $(1,1)$. Using the histologic analysis of the samples as the reference method, each experimental point in the phase plane $(K, M)$ in Fig. 2 can be associated with a certain type of cancer or healthy tissue.

For selecting classes in the phase space $(K, M)$ we used the canonical linear discriminant analysis [25]. The quality of approach was characterised by its sensitivity and specificity. Varying the class priorities one can shift the boundary line, achieving the required proportion of sensitivity and specificity.

The sequential discriminant analysis appeared to be the most efficient one (Table 1):

1. Selection of nevus (straight line 1) with the sensitivity $86 \%$ and specificity $96 \%$ from all cancer types - Class I.
2. Selection of basalioma (straight line 2) with the sensitivity $90 \%$ and specificity $94 \%$ from all studied malignant tumors - Class II.

3. Differentiation of melanoma (Class III) and squamous cell carcinoma (class IV) - the straight line 3.

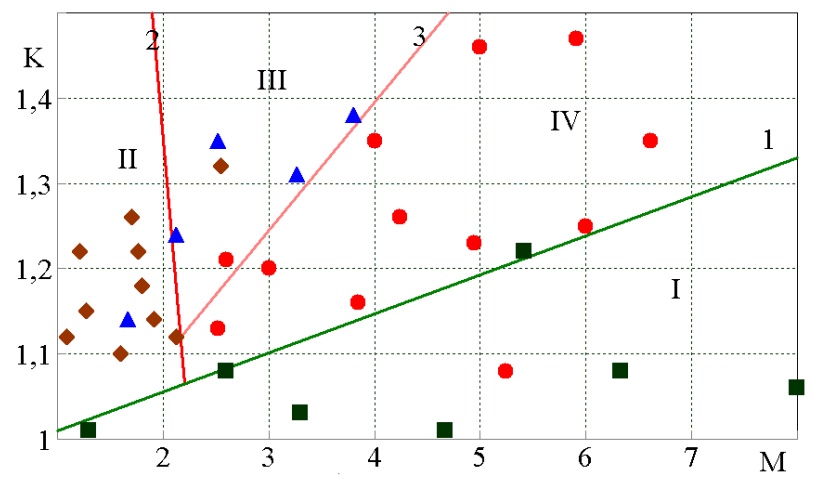

Fig. 2 Phase diagram for different types of skin cancers: nevus (square), basalioma (rhombus), squamous cell carcinoma (triangle), melanoma (circle). Class I - the region of nevus and normal skin, Class II - the region of basalioma, Class III-the region of squamous cell carcinoma, and Class IV - the region of melanoma.

\section{Tumor boundary inedtification}

The proposed approach can be used not only for determining the cancer type, but also for rapid identification of its boundary. The latter is of particular importance when preparing for surgical operation, or observing in the course of chemotherapy treatment.

Figure 3 presents a typical pattern of distributions of optical characteristics in the phase plane $(K, M)$ recorded at the points $(x, y)$ along the tumour section for basalioma (a) and melanoma (b). In all experiments the scanning was performed starting from the region of definitely healthy skin, passing the tumor and reaching the healthy skin or the centre of inhomogeneity (in the case of sample excision). The scanning step is $1 \mathrm{~mm}$, the diameter of focal spot of the optical radiation on the skin surface about $1.5 \mathrm{~mm}$. The spectrum was recorded at $16-25$ points for each experimental series.

It is seen that for basalioma the points corresponding to healthy skin lie either in the region belonging to class I (benign tumors) or near the point $(1,1)$ in the region of Class II. As we move towards the tumour centre, the corresponding points in the phase plane move up along the axis $K$, while the value of $M$ remains unchanged within the measurement error. This corresponds to the fact that for basalioma the melanin concentration changes weakly in comparison with that of the healthy skin adjacent to the tumour. On the contrary, for melanoma the increase of melanin concentration and density of capillaries is typical, as we move towards the tumour centre, which leads to the prolate distribution of the tumour points in the phase plane region belonging to Class IV. And the points corresponding to the healthy tissue are localised beyond the region of class IV, near 
Table 1 Neoplasms classification.

\begin{tabular}{|c|c|c|c|c|c|c|}
\hline \multirow{2}{*}{ Stage } & \multirow{2}{*}{ Samples } & \multirow{2}{*}{ Histology } & \multicolumn{2}{|c|}{ Backscattering } & \multirow{2}{*}{ Sensitivity } & \multirow{2}{*}{ Specificity } \\
\hline & & & Region A & Region B & & \\
\hline \multirow{2}{*}{1} & A. Nevus & 7 & 6 & 1 & \multirow{2}{*}{$86 \%$} & \multirow{2}{*}{$96 \%$} \\
\hline & B. Other neoplasms & 27 & 1 & 26 & & \\
\hline \multirow{2}{*}{2} & A. Basalioma & 10 & 9 & 1 & \multirow{2}{*}{$90 \%$} & \multirow{2}{*}{$94 \%$} \\
\hline & B. Melanoma, squamous cell carcinoma & 17 & 1 & 16 & & \\
\hline \multirow{2}{*}{3} & A. Melanoma & 12 & 10 & 2 & \multirow{2}{*}{$83 \%$} & \multirow{2}{*}{$80 \%$} \\
\hline & B. Squamous cell carcinoma & 5 & 1 & 4 & & \\
\hline
\end{tabular}
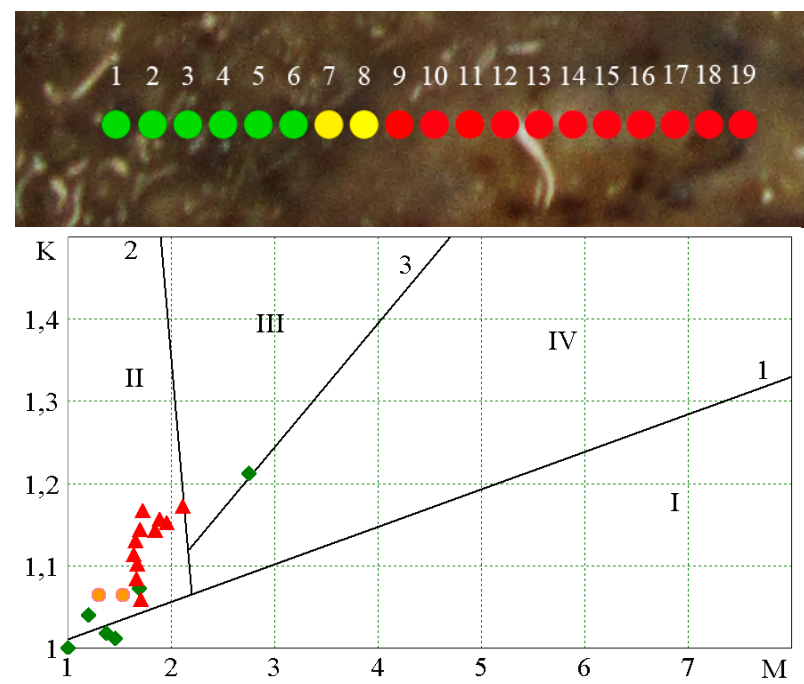

a) Basal cell carcinoma
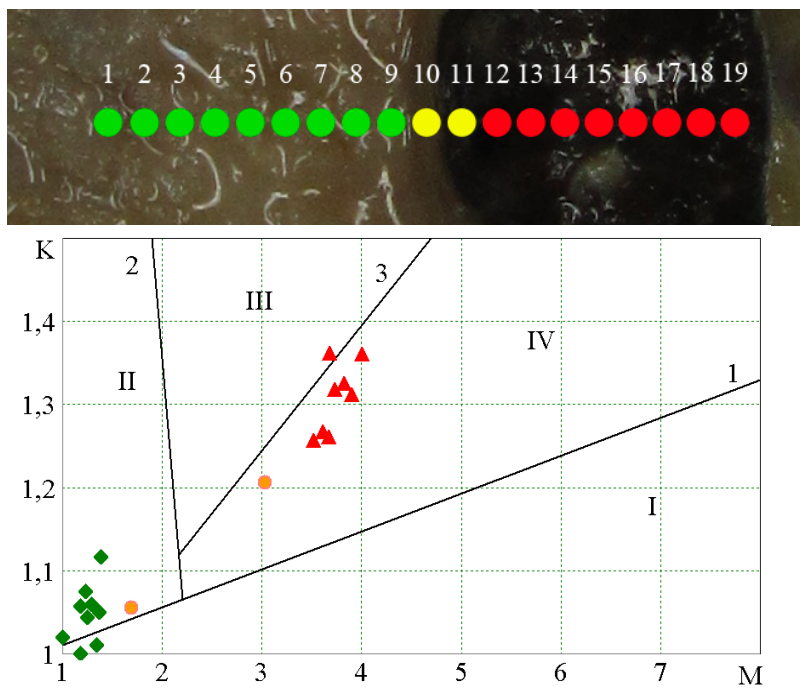

b) Melanoma

Fig. 3 Distribution of scanning point in the two-dimensional plane of spectral indices $(K, M)$ for basal cell carcinoma (a) and melanoma (b). The rest notations correspond to Fig. 2.

the line 1, separating the classes of malignant and benign tumors.

\section{Conclusion}

We propose a method for detection and classification of skin pathologies, based on differential analysis of backscattered spectral intensities with the individual features of the human skin optical characteristics taken into account. The approach allows differentiation of benign tumors from the malignant ones with high sensitivity and specificity. However, the classes of malignant tumors partially overlap in the phase plane of normalised scattering indices $(\mathrm{K}, \mathrm{M})$. This fact reduces the sensitivity of the malignant tumour type identification. For example, for melanoma the sensitivity is limited by $83 \%$ and the specificity by $80 \%$. Thus, it was achieved the increase $7 \%$ for sensitivity and $5 \%$ for specificity in comparison with known methods [26]. The main reason for such enhancement of sensitivity and specificity is connected with better accuracy of two-dimensional phase space analysis and spectral normalization of optical indexes on their values in neighboring healthy skin, which exclude the influence of individual characteristics of skin associated with a sunburn or skin phenotype.
Generally, one can assert that the method of differential scattering allows the localisation of tumor boundary with the error about $1 \mathrm{~mm}$, limited by the focusing spot size and the region of efficient scattering of the probing radiation.

With low probability of missing the disease and the personalisation of the analysis, this approach can be used in mass examinations of population. And the capability of precise boundary determination is important for both the treatment planning and the treatment efficiency skin cancer.

\section{Acknowledgments}

This research was supported by the Ministry of Education and Science of the Russian Federation. 